\title{
Exposure to the World Trade Center Disaster and 9/11-Related Post-Traumatic Stress Disorder and Household Disaster Preparedness
}

Lisa M. Gargano, PhD, MPH; Kimberly Caramanica, MPH; Sarah Sisco, MPH, MSSW;

Robert M. Brackbill, PhD, MPH; and Steven D. Stellman, PhD, MPH

\section{ABSTRACT}

Objective: In a population with prior exposure to the World Trade Center disaster, this study sought to determine the subsequent level of preparedness for a new disaster and how preparedness varied with population characteristics that are both disaster-related and non-disaster-related.

Methods: The sample included 4496 World Trade Center Health Registry enrollees who completed the Wave 3 (2011-2012) and Hurricane Sandy (2013) surveys. Participants were considered prepared if they reported possessing at least 7 of 8 standard preparedness items. Logistic regression was used to determine associations between preparedness and demographic and medical factors, 9/11-related post-traumatic stress disorder (PTSD) assessed at Wave 3, 9/11 exposure, and social support.

Results: Over one-third (37.5\%) of participants were prepared with $18.8 \%$ possessing all 8 items. The item most often missing was an evacuation plan (69.8\%). Higher levels of social support were associated with being prepared. High levels of 9/11 exposure were associated with being prepared in both the PTSD and non-PTSD subgroups.

Conclusions: Our findings indicate that prior 9/11 exposure favorably impacted Hurricane Sandy preparedness. Future preparedness messaging should target people with low social support networks. Communications should include information on evacuation zones and where to find information about how to evacuate. (Disaster Med Public Health Preparedness. 2015;9:625-633)

Key Words: World Trade Center, preparedness, post-traumatic stress disorder, Hurricane Sandy

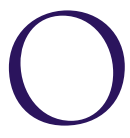
ne of the most effective ways to mitigate the effects of a disaster is through proper household emergency preparedness. ${ }^{1,2}$ Official recommendations from the American Red Cross (ARC), the US Federal Emergency Management Agency (FEMA), and the US Department of Homeland Security (DHS) call for the assembly of a disaster preparedness kit containing essential supplies such as food, water, and prescription medications in sufficient quantities for 3 days for each member of the household, as well as having an established disaster plan. Despite these recommendations, only $25 \%$ of US households currently adhere to the minimum ARC, FEMA, and DHS preparedness guidelines, including a 3-day supply of food, water, and medication and a written household evacuation plan. ${ }^{3}$

Social, economic, and demographic characteristics influence the likelihood that an individual or household has sufficiently prepared for a disaster. Older age and higher education and income are associated with increased emergency preparedness in US and international samples. ${ }^{4-10}$ Higher socioeconomic status variables (eg, higher income, home ownership) have been consistently associated with completion of disaster preparedness tasks such as storing food, water, or first-aid supplies. ${ }^{4,6,8-10}$ In multiple large studies, racial/ethnic minority status was associated with lower scores on multi-item scales assessing possession of specific disaster items. ${ }^{6,7,9,11}$ Whereas minorities tend to be less prepared before a disaster, these groups may demonstrate greater increases in preparedness after an event. ${ }^{10,12,13}$ Other studies have noted that having medical vulnerabilities such as disabilities and chronic medical conditions correlates with a lack of disaster preparedness. Persons with disabilities are less likely to have emergency supplies on hand and to evacuate following a mandatory evacuation order. ${ }^{11,14}$ Similarly, persons with perceived poor general health are less prepared for disasters and are less likely to have an emergency communication plan than are those who perceive their health as being excellent. ${ }^{7,14,15}$ Strine et al found that people with inadequate social and emotional support are less likely to be prepared. ${ }^{16}$ 
Studies have also found that persons with serious mental illness or frequent mental distress are less likely to be prepared. $^{7,16}$

The present study examined whether prior trauma affects personal preparedness for future disasters. In the literature, the association between prior disaster experience and preparedness is not clearly established. Some studies show a correlation between prior disaster experience and increased preparedness. ${ }^{8,17-20}$ Intensity of exposure to the September 11, 2001 (9/11), World Trade Center attack as well as greater lifetime exposure to traumatic events were both shown to be predictive of greater personal preparedness in a random sample of New York City adults. ${ }^{17}$ However, other studies showed no difference in preparedness after a major disaster. $^{21-23}$ As part of the 2009 National Hurricane Survival Initiative, a survey found that $66 \%$ of people living in Atlantic and Gulf Coast states, areas with hurricane exposure, had no hurricane survival kit and 55\% had no family disaster plan. ${ }^{22}$ There are several proposed explanations for this finding. First, this may be attributed to a ceiling effect of an already high level of preparedness among the population. ${ }^{21}$ Second, having experienced previous disasters may create an optimistic bias, where individuals minimize the need for extensive preparation. ${ }^{20}$ In addition, when each preparedness task (eg, planning, stockpiling) received separate consideration, experience was not clearly related to both types of preparedness actions. ${ }^{13,24}$ After the 2005 London subway bombings, Page et al found that emergency planning increased from $51 \%$ to $90 \%$, but the number of respondents who gathered emergency supplies before and after the event did not increase. ${ }^{13}$

Disaster survivors who experience higher levels of property loss and psychological distress may be more attentive to news reports and have a better understanding of the destructive consequences of disasters and therefore be more prepared..$^{20,25-27}$ Sattler et al found that among participants with hurricane experience, distress as a result of the hurricane accounted for a significant portion of preparation variance. ${ }^{20}$ Another study found that people with stronger negative memories of past disasters engaged in more disaster preparedness. ${ }^{25}$ Preparedness for earthquakes and hurricanes has been associated with the amount and cost of damage experienced during a previous disaster. ${ }^{26,27}$ Nevertheless, few studies have assessed the ability of individuals seriously affected by one disaster to prepare for subsequent events.

On October 29, 2012, Hurricane Sandy struck a heavily populated area of the northeastern United States. It was the largest and second costliest hurricane in US history, and resulted in numerous deaths and an estimated $\$ 50$ to $\$ 65$ billion in damages. ${ }^{28,29}$ Sandy occurred 11 years after the terrorist attacks of 9/11, which killed nearly 3000 people, injured thousands more, and had profound effects on the psychological status of area residents. ${ }^{30,31}$ Hurricane Sandy thus subjected thousands of people to a second life-threatening disaster in a relatively short period of time. It also occurred shortly after the World Trade Center Health Registry's (Registry) completion of a follow-up health survey of over 43,000 enrollees in an ongoing cohort study of 9/11 responders and survivors, over $60 \%$ of whom resided in the New York City area. This provided the opportunity to study the effects of Hurricane Sandy in a population with well-documented exposure to a prior disaster.

In the present study, we used data from the Registry to assess, in a population with prior exposure to the $9 / 11$ disaster which had well-recognized, serious health and life consequences, the subsequent level of preparedness for a new disaster (Hurricane Sandy). In addition, we examined (1) the impact of 9/11related post-traumatic stress disorder (PTSD) on a person's ability to prepare, (2) the role of social support in preparedness among people exposed to $9 / 11$, and (3) the relationship between preparedness and knowledge of evacuation zones for Hurricane Sandy.

\section{METHODS \\ Data Source}

The Registry, a longitudinal cohort study of 71,431 people exposed to the 9/11 disaster in New York City, provided the sampling pool for this study. Details on recruitment methods and Registry findings have been published elsewhere. ${ }^{30,32}$ Briefly, Registry enrollees belong to 1 or more of 5 eligibility groups: rescue/recovery workers and volunteers; lower Manhattan residents; area workers; passersby; and schoolchildren and staff. There were 2 modes of enrollment into the Registry: list-identified enrollees (30\%) were actively recruited from lists provided by governmental agencies, organizations, and employers, whereas self-identified enrollees (70\%) contacted the Registry via phone or pre-registered on a website in response to intensive outreach efforts. ${ }^{32}$ The Wave 1 baseline survey (2003-2004) included 68,805 adults and elicited information on 9/11-related exposures and indicators of physical and mental health. ${ }^{32}$ The Wave 2 (2006-2007) and Wave 3 (2011-2012) surveys obtained additional information on 9/11-related exposures and provided an update on health status $(46,602$ [68\%] for Wave 2 and 43,134 [63\%] for Wave 3 of the original adult enrollees). ${ }^{30}$ Since Hurricane Sandy occurred shortly after completion of the third wave of Registry data collection, selection for the Sandy substudy was restricted to those enrollees who had completed the Wave 3 survey and resided in the tri-state area (New York, New Jersey, and Connecticut). Approximately 5 months after Hurricane Sandy (March 2013), selected enrollees were contacted via mail or e-mail with a letter that described the study and invited their participation. ${ }^{33}$ Those with valid e-mail addresses were invited to participate in a web survey ( $\mathrm{n}=7367$ ). Three weeks after the launch of the web-based portion of data collection, paper surveys were mailed to study subjects who did not have a valid e-mail address $(n=1503)$ 
or who did not complete the web-based survey. Multiple mail and e-mail reminders were sent to nonrespondents, as well as multiple rounds of paper questionnaires. At the close of data collection (November 2013), 4558 surveys had been completed $(51.4 \%$ response rate). The study protocol was approved by the New York City Department of Health and Mental Hygiene's Institutional Review Board.

\section{Study Sample}

The study sample included all 4558 people who submitted surveys except the 62 people with incomplete information on Hurricane Sandy preparedness, resulting in a final sample size of 4496 .

\section{Dependent Variable}

The study outcome was derived from responses to the following question, "Which of the following did your household have prepared before Sandy happened? Don't include things you did during or after the storm." Similar to a previous report, respondents were deemed to be "prepared" if they had no less than 1 item missing of the 8 preparedness action items. ${ }^{4}$ This definition was chosen among different possible options because being prepared is an ongoing process, rather than a destination. ${ }^{34}$ Allowing for one missing item on the preparedness checklist acknowledged the reality of preparedness as a process and the ambiguity inherent in the lack of a universal definition of preparedness, while also allowing those clearly in the process of becoming prepared to be included among the prepared. This methodology also allowed us to refrain from attempting to define an interval measure for preparedness derived from weighting the relative importance of the 8 disparate preparedness checklist items to overall preparedness. ${ }^{4}$ The 8 preparedness checklist items were as follows:

- Supply of food

- Three-day supply of water

- First aid kit

- Battery-operated radio

- Needed medications

- Flashlight

- Personal care and hygiene items (eg, toothpaste, diapers, and toilet paper)

- Evacuation plan

\section{Demographic Variables}

Ethnic and racial groups were categorized as Hispanic, white non-Hispanic, black non-Hispanic, Asian, and other. Age at the time of the Hurricane Sandy survey was categorized as 18 to 44 years, 45 to 64 years, and 65 years or older. Annual household income in 2010 was categorized as less than or equal to $\$ 75,000$ and greater than $\$ 75,000$.

\section{Medical Factors}

Individuals with medical conditions may be more vulnerable during disasters because of their dependence on prescription medications, potential need for additional medical attention at a shelter, potential reliance on electricity for medical equipment or refrigeration of medications, or their hindered mobility, which may make complying with evacuation orders difficult. Victims displaced by Hurricane Katrina frequently required acute or preventive medical care at shelters, including prescription medications and durable medical goods. ${ }^{35,36}$ Self-reported physician-diagnosed asthma, diabetes, and coronary heart disease at Wave 3 were therefore included as medical risk factors in the analysis. ${ }^{35-38}$

\section{9/11-Related Post-Traumatic Stress Disorder}

Probable PTSD was assessed at Wave 3 by using the PTSD Checklist-Civilian Version (PCL). The PCL is a self-reported, 17 -item scale corresponding to the criteria in the Diagnostic and Statistical Manual of Mental Disorders, 4th edition (DSMIV), ${ }^{39,40}$ that is commonly used in epidemiologic research. Selected symptoms were queried as specific to $9 / 11$ and all were current (within the past 30 days). Psychometric properties of the PCL used in this manner in other settings and with other traumatized populations have reported sensitivity ranging from 0.94 to 0.97 , specificity from 0.86 to 0.99 , positive predictive value from 0.70 to 0.97 , and diagnostic efficiency from 0.83 to $0.96 .^{39-41}$ Because studies have shown that the diagnostic efficiency of the PCL is improved when using a cutoff of 44 as opposed to $50,{ }^{39}$ in this study, as in previous Registry studies, ${ }^{30,32,42,43}$ enrollees with a PCL score of 44 or greater at Wave 3 were considered to have probable PTSD.

\section{Social Support}

An index of social support was created by summing 5 Wave 3 items that pertain to perceived or received support, including how often someone is available to take the respondent to the doctor, have a good time with them, hug them, prepare meals if they are unable to do it themselves, and understand their problems. The five items were scored on a scale of 0 (none of the time) to 4 (all of the time). Items were summed with the total index ranging from 0 to 20 . Scores were classified as low or no social support (0-6), medium (7-11), high (12-15), and very high social support (16-20). This social support scale has been used in previous Registry studies and is a strong predictor of unmet mental health care need. ${ }^{44}$

\section{9/11 Exposure}

Exposure to $9 / 11$ was defined with a 12-item summary measure used in previous Registry reports ${ }^{45}$ that is modeled after work by Adams et al. ${ }^{46}$ On the basis of the score distribution, exposure was categorized as none/low (0-1 experiences), medium (2-3), high (4-5), and very high (6 or more). This scale covers several dimensions of 9/11-related exposure, and each item has been shown to be associated with physical or mental health outcomes in previous Registry studies. ${ }^{30,32}$ 


\section{Knowledge of Evacuation Zone}

The Hurricane Sandy survey queried whether participants knew if they lived in an evacuation zone prior to Sandy. These zones are part of New York City's hurricane contingency plan and are areas of the city that may be inundated by storm surge or isolated by storm surge waters. ${ }^{47}$ There are 6 zones of varying levels of likelihood of inundation, which can be identified for a given address with a single web query. Response options were, "Yes, I knew I lived in an evacuation zone," "Yes, I knew I did not live in an evacuation zone," and "No, I did not know whether I lived in an evacuation zone."

\section{Statistical Analysis}

Univariate analysis was conducted to describe preparedness in the study population and demographic, medical, mental health, and 9/11 exposure factors. Cochran-Armitage tests were used to detect trends between social support and preparedness and 9/11-exposure category and preparedness in the full sample and when stratified by PTSD. Using logistic regression, unadjusted and adjusted odds ratios (aORs) were calculated to estimate the strength of the association between selected covariates and preparedness. According to the results of preliminary analyses, the adjustment variables were as follows: sex, age group, race/ethnicity, household income in 2010, asthma, diabetes, coronary heart disease, social support, 9/11 exposure, 9/11-related PTSD at Wave 3, and knowledge of evacuation zone. Previous Registry studies have shown that PTSD acts as a modifier in several 9/11-related health outcomes; ${ }^{43,48-50}$ therefore $9 / 11$-related PTSD was examined as a potential modifier of the relationship between $9 / 11$ exposure and preparedness. The final model assessed the association between $9 / 11$ exposure and preparedness stratified by $9 / 11$. related PTSD status. All analyses were conducted by using SAS 9.2 (Cary, NC).

\section{RESULTS}

\section{Respondent Characteristics}

As shown in Table 1, the majority of the respondents were male $(56.0 \%), 45$ to 64 years of age $(62.9 \%)$, non-Hispanic white $(71.1 \%)$, and had a 2010 annual income of greater than $\$ 75,000$ (57.7\%). With respect to medical conditions, the highest proportion of respondents reported having asthma (22.4\%). Less than one-fifth (19.1\%) of respondents had probable PTSD at Wave 3. Only 15.0\% reported having none/ low social support. The majority knew whether they lived $(35.5 \%)$ or did not live $(40.3 \%)$ in an evacuation zone. Almost three-quarters (73.1\%) had at least two 9/11 exposures, and $10.5 \%$ had very high $9 / 11$ exposure (6 or more exposures).

On the basis of the study criteria, a total of 1684 respondents were considered "prepared" for the arrival of Hurricane Sandy $(37.5 \%)$. A total of 845 respondents $(18.8 \%)$ reported having all 8 preparedness items (Table 2). Only $4.8 \%$ reported that they did not prepare anything before the storm. A flashlight was the most commonly reported item $(91.7 \%)$, followed by personal care and hygiene kits $(80.5 \%)$ and nonperishable food or snacks (76.8\%) (Table 3). The item that respondents were least likely to have prepared was an evacuation plan $(30.2 \%)$.

\section{Factors Associated With Preparedness}

Preparedness varied with race/ethnicity, from $24.6 \%$ of Asians to $40.3 \%$ of non-Hispanic whites (Table 1). Fewer respondents with an income of $\$ 75,000$ or less were prepared $(33.3 \%)$ compared to those with income of greater than $\$ 75,000$ (40.5\%). Of those with 9/11-related PTSD, 39.9\% were prepared compared with $37.0 \%$ of those without PTSD. Only $28.2 \%$ of those with the lowest social support score were prepared compared to $42.8 \%$ in the group with the highest social support score. Those who knew they lived in an evacuation zone had the highest frequency of being prepared $(42.5 \%)$ compared to $27.1 \%$ who did not know if they lived in an evacuation zone. Respondents with very high 9/11 exposure (6 or more exposures) had the largest frequency of preparedness (47.6\%) compared to $31.2 \%$ of the respondents with none/low $9 / 11$ exposure (0-1 exposure) (Table 1).

In unadjusted analyses, sex, age, race/ethnicity, income, social support, knowledge of living in an evacuation zone, and 9/11 exposure were all significantly associated with Hurricane Sandy preparedness (Table 1). In adjusted analyses, sex and income were no longer significant and only Asians compared to non-Hispanic whites were significantly less likely to be prepared. Of the medical factors, only asthma was significant in the adjusted model; those with asthma were $20 \%$ more likely to be prepared than were those without asthma (aOR: 1.2; $95 \%$ confidence interval [CI]: 1.0-1.4).

Compared to those who did not know whether they lived in an evacuation zone, those who knew they lived in an evacuation zone were almost twice as likely to be prepared $(\mathrm{aOR}$ : 1.9; 95\% CI: 1.6-2.3); those who knew they did not live in an evacuation zone were also more likely to be prepared (aOR: 1.7; 95\% CI: 1.4-2.0). 9/11-related PTSD was not significantly associated with preparedness in either the unadjusted or the adjusted analysis. There was a relationship between social support and Hurricane Sandy preparedness in the adjusted model $(P<0.0001)$. Compared to those with none/low social support, those with high social support were 50\% more likely to be prepared (aOR: 1.5; 95\% CI: 1.1-1.8) and those with very high social support were almost twice as likely to be prepared (aOR: 1.9; 95\% CI: 1.5-2.4) (Table 1).

With respect to 9/11 experiences, there was also a strong relationship between 9/11 exposure and preparedness. Compared to those with none/low 9/11 exposure (0-1 exposure), those who were most highly exposed (6 or more exposures) were nearly twice as likely to be prepared ( $\mathrm{aOR}$ : 1.8; 95\% CI: 1.4-2.2) (Table 1). 
Study Population and Association Between Disaster Preparedness and Selected Demographic Characteristics, Medical Factors, Mental Health Characteristics, and 9/11 Exposures ${ }^{a}$

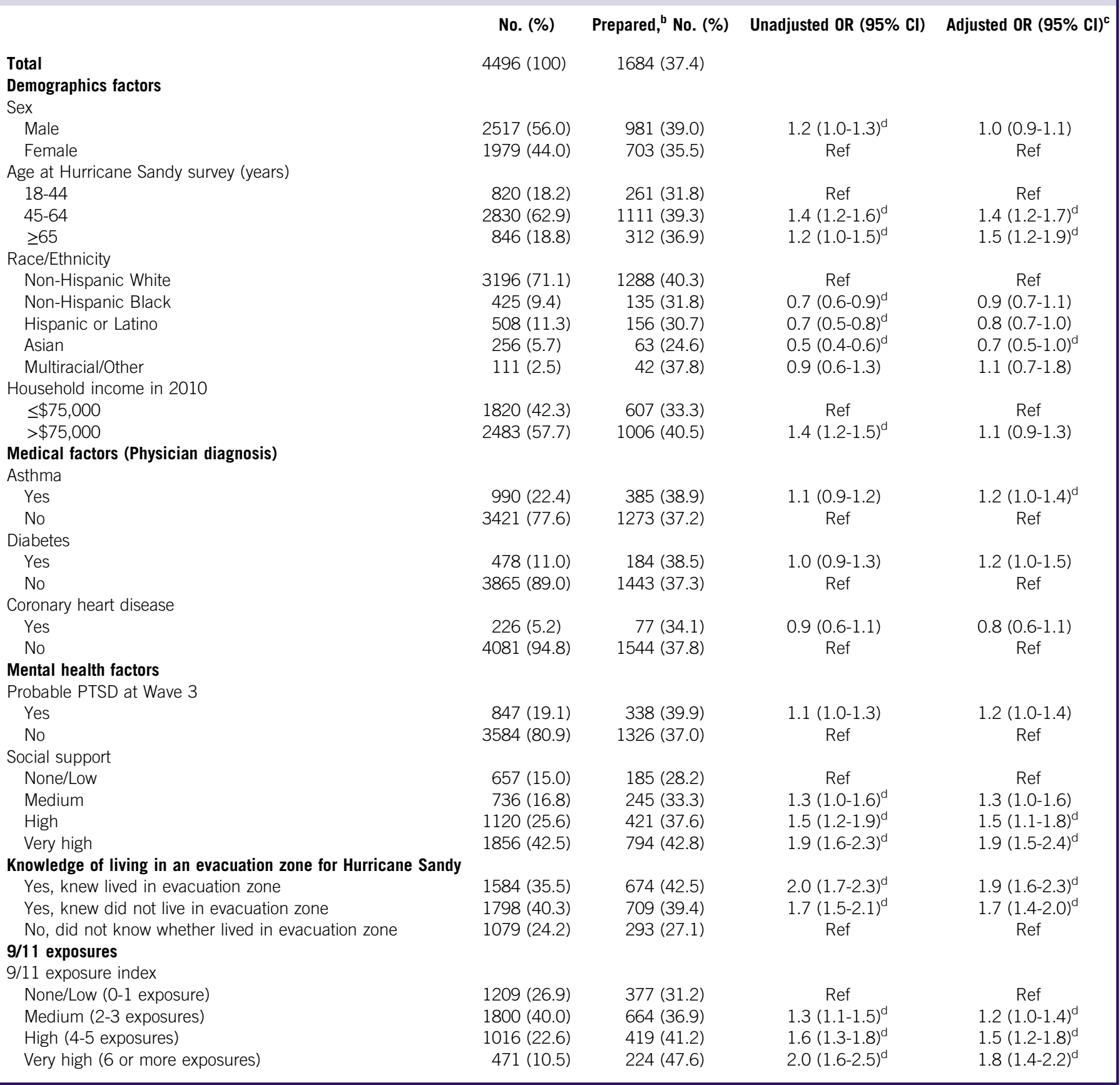

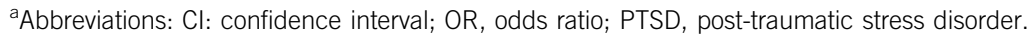

${ }^{\mathrm{b}}$ Reported having at least 7 of 8 preparedness items.

${ }^{\mathrm{c}}$ Adjusted for sex, age group, race/ethnicity, household income, asthma, diabetes, coronary heart disease, social support, World Trade Center exposure, PTSD, and knowledge of evacuation zone.

${ }^{\mathrm{d}} P \leq 0.05$.

\section{Association of 9/11 Exposures and Preparedness by 9/11-Related PTSD Status}

Although nearly 1 in 5 study participants had probable PTSD at Wave 3, this condition was not predictive of preparedness
(Table 1). Nevertheless, it was of interest to determine whether the experiences of 9/11 exposure were associated with preparedness, and if so, whether that association differed among those with and without 9/11-related PTSD. Table 4 


\section{TABLE 2}

\begin{tabular}{|lr|}
\hline Distribution of Total Preparedness Items in the Sample \\
Items checked & No. (\%) \\
None of the above & $214(4.8)$ \\
1 & $121(2.7)$ \\
2 & $199(4.4)$ \\
3 & $329(7.3)$ \\
4 & $510(11.3)$ \\
5 & $639(14.2)$ \\
7 & $800(17.8)$ \\
8 & $839(18.7)$ \\
\hline
\end{tabular}

TABLE 3

\section{Frequencies of Preparedness Items Checked}

Which of the following did you have prepared

before Hurricane Sandy happened?

Yes, No. (\%)

Flashlight

Personal care and hygiene items

Nonperishable food or snacks

3 days' worth of drinking water per person

Needed medicines

First aid kit

Battery operated radio

Evacuation plan

None of the above

$4121(91.7)$

$3620(80.5)$

$3454(76.8)$

$2947(65.5)$

$2921(65.0)$

2917 (64.9)

$2835(63.1)$

$1359(30.2)$

$214(4.8)$

\section{TABLE 4}

Association Between Disaster Preparedness and 9/11 Exposures Stratified by 9/11-Related PTSD at Wave $3^{\mathrm{a}}$

\begin{tabular}{|lcc} 
& \multicolumn{2}{c}{ Adjusted $\left.\mathbf{O R}^{\mathbf{b}} \mathbf{( 9 5 \%} \mathbf{~ C I}\right)$} \\
\cline { 2 - 3 } 9/11 Exposure Index & $\begin{array}{c}\text { Probable PTSD } \\
(\mathbf{n}=\mathbf{8 4 7})\end{array}$ & $\begin{array}{c}\text { No Probable } \\
\text { PTSD }(\mathbf{n}=\mathbf{3 5 8 4})\end{array}$ \\
None/Low (0-1 exposure) & $1.3(0.8-2.0)$ & Ref \\
Medium (2-3 exposures) & $1.5(1.1-2.0)^{\mathrm{C}}$ & $1.2(1.0-1.5)^{\mathrm{C}}$ \\
High (4-5 exposures) & $1.7(1.2-2.3)^{\mathrm{C}}$ & $1.5(1.2-1.8)^{\mathrm{C}}$ \\
Very high (6 or more exposures) & $2.0(1.4-2.9)^{\mathrm{C}}$ & $1.8(1.4-2.4)^{\mathrm{C}}$ \\
\hline
\end{tabular}

${ }^{a}$ Abbreviations: $\mathrm{Cl}$ : confidence interval; OR, odds ratio; PTSD, post-traumatic stress disorder.

${ }^{\mathrm{b}}$ Adjusted for sex, age group, race/ethnicity, household income, asthma, diabetes, coronary heart disease, social support, World Trade Center exposure, and knowledge of evacuation zone.

${ }^{\mathrm{c}} P \leq 0.05$.

shows the aORs for disaster preparedness in relation to 4 levels of the 9/11 exposure index, using none/low $(0-1$ exposure) as the common reference for those with and without 9/11-related PTSD. aORs increased with increasing degree of $9 / 11$ exposure $(P$ value $<0.0001)$. The $O R$ at each level of exposure was slightly higher among people with
PTSD than without, but these differences were not significant. Compared to those who had none/low 9/11 exposure (0-1 exposure) and no PTSD, those with medium ( $2-3$ exposures) and high (4-5 exposures) exposure and 9/11related PTSD were 50\% (aOR: 1.5; 95\% CI: 1.1-2.0) and 70\% (aOR: 1.7; 95\% CI: 1.2-2.3) more likely to be prepared, respectively. Those with very high exposure (6 or more exposures) and 9/11-related PTSD were twice as likely to be prepared (aOR: 2.0; 95\% CI: 1.4-2.9).

\section{DISCUSSION}

In this study we found that a lower proportion (18.8\%) of Registry enrollees had all 8 preparedness items when compared to the general US population $(25 \%))^{3}$ Respondents with very high 9/11 exposure had the highest frequency of preparedness compared to the respondents with none/low 9/11 exposure. Moreover, 9/11 exposure was associated with preparedness irrespective of 9/11-related PTSD status; however, the association was slightly stronger among those with 9/11-related PTSD than among those without PTSD. Findings on the association between previous disaster experience and preparedness are mixed. While several studies have shown that distress over a prior disaster experience is associated with increased preparedness, ${ }^{20,25-27}$ a study conducted after the 2005 London subway bombing found that the degree of psychological distress from a prior disaster experience does not clearly predict preparedness behaviors. ${ }^{13}$ Faupel and Styles found that psychological stress correlated with increased household disaster planning. ${ }^{51}$ Ultimately, it is an individual's perceived risk of future disasters that impacts the likelihood of preparedness. Higher perceived risk has been associated with greater rates of personal preparedness activities. ${ }^{52,53}$ This is likely due to a complex relationship between sociodemographic and medical characteristics, prior disaster experiences, temporal spacing between experiences, and negative disaster outcomes. Regardless of a negative outcome (PTSD), the 9/11 experiences of enrollees may have been so impactful on their perception of risk that it led to an increase in their own preparedness activity.

We found that having strong social support was positively associated with preparedness. Previous studies have shown that having high social capital or social support are associated with increased preparedness. ${ }^{18,26}$ Considerable evidence also indicates that people who have social support are generally in better mental and physical health than are those without. ${ }^{7,54,55}$ The more confident people are about their ability to successfully respond to an emergency, the more likely they are to engage in preparedness behaviors. Peers and families are valued means to create self-efficacy and confidence; people are more likely to prepare if those around them believe in being prepared. ${ }^{56-58}$ This means that people who experience strong social support may have the importance of preparation conveyed to them personally, as well as reinforced numerous 
times. Although people can learn how to prepare from media sources, information alone is rarely sufficient to change behavior. If social support networks reiterate preparedness, specifically demonstrating investment in a person's well-being before, during, and after an emergency, this may elevate preparedness as important enough to undertake. ${ }^{26}$

Similar to other studies, we found that individuals are less likely to create an evacuation plan than to assemble a supply kit. ${ }^{4,14,16} \mathrm{We}$ also found that older age and higher socioeconomic status are associated with increased emergency preparedness; this finding has been observed in other US and international studies. ${ }^{4,6,9}$ Similar to our findings, several large studies found that racial/ethnic minority status is associated with lower preparedness. ${ }^{6,7,9,11}$

Although it was encouraging to find that less than one-quarter of participants did not know whether they lived in an evacuation zone, we observed that those participants were less likely to be prepared than were those who knew whether they did or did not live in an evacuation zone. It is possible that individuals who are knowledgeable about their evacuation zone also tend to be aware of what preparedness actions need to be completed. This finding has important implications for preparedness communications and response operations; future campaigns should consider combining information on evacuation zones ${ }^{59}$ with preparedness messaging and instructions on how to establish an evacuation plan.

\section{Strengths and Limitations}

This study is subject to several limitations. Although the 8 objective measures of Hurricane Sandy preparedness included in the analysis were derived from previously published studies, ${ }^{4,16}$ the mere possession of these items does not suggest that they are located in a "go-bag" or that they are current and functional. A future refinement of preparedness questions to include a focus on stockpiling goods and materials specifically for a future disaster is needed. Sensitivity analyses were performed on a preparedness outcome with no missing items and with no more than 2 missing items and the associations were similar in magnitude to our a priori definition of being prepared. We did not measure whether participants had any knowledge of how to prepare. Future research should assess knowledge of how to prepare in addition to questions about items on hand. Because data were collected 5 to 12 months after the event, our findings may be subject to recall bias. In addition, all data collected were self-reported. The absence of an observable association between PTSD and preparedness could be attributed to the time between data collection for the Wave 3 and Sandy surveys. Furthermore, the findings presented may not be generalizable to other populations because the sample was limited to those who experienced the 9/11 disaster and may not be representative of the entire population impacted by Hurricane Sandy or applicable to those with other previous disaster experience. Despite these limitations, the Registry provided a unique opportunity to rapidly study a large number of people previously exposed to a disaster and their level of preparedness for a subsequent disaster. Additionally, this study assessed preparedness for an actual disaster event and not preparedness in general with no imminent disaster. For future storms, framing of preparedness messages can be based on findings from this study, especially targeting those with weak or no social support and encouraging all to have specific evacuation plans.

\section{CONCLUSIONS}

Understanding how previous disaster experiences and possible mental health outcomes affect preparedness can be important for future preparedness planning. Future research is needed to develop messaging techniques that encourage supply-gathering behaviors in general, but especially evacuation planning. Emergency planners should consider developing preparedness messaging to target providers and community outreach groups who may have contact with vulnerable populations and individuals characterized by low social support (eg, undocumented immigrants, homebound older adults) who could benefit from increased preparedness awareness.

\section{About the Authors}

World Trade Center Health Registry, New York City Department of Health and Mental Hygiene, Long Island City, New York (Drs Gargano, Brackbill, and Stellman and Ms Caramanica); Office of Emergency Preparedness and Response, New York City Department of Health and Mental Hygiene, Long Island City, New York (Ms Sisco); and Department of Epidemiology, Mailman School of Public Health, Columbia University, New York, New York (Dr Stellman)

Correspondence and reprint requests to Lisa M. Gargano, PhD, MPH, 42-09 28th Street, 7th Floor, Long Island City, NY 11101 (e-mail: lgargano1@health.nyc.gov).

\section{Acknowledgments}

This study was supported by Cooperative Agreement Numbers 5U50/ OH009739 and 1E11/OH009630 from the National Institute for Occupational Safety and Health (NIOSH) of the Centers for Disease Control and Prevention (CDC); U50/ATU272750 from the Agency for Toxic Substances and Disease Registry (ATSDR), CDC, which included support from the National Center for Environmental Health, CDC; and by the New York City Department of Health and Mental Hygiene (NYC DOHMH). Its contents are solely the responsibility of the authors and do not necessarily represent the official views of NIOSH-CDC.

Published online: June 15, 2015.

\section{REFERENCES}

1. Falkiner L. Impact analysis of the Canadian Red Cross Expect the Unexpected Program. 2003. http://www.redcross.ca/cmslib/general/imact_ analysis_full_version.pdf. Accessed March 10, 2015.

2. Keim ME. Building human resilience: the role of public health preparedness and response as an adaptation to climate change. Am J Prev Med. 2008;35(5):508-516.

3. DeBastiani SD, Strine TW. Household preparedness for public health emergencies - 14 states, 2006-2010. MMWR Morb Mortal Wkly Rep. 2012;61(36):713-719. 
4. Ablah E, Konda K, Kelley CL. Factors predicting individual emergency preparedness: a multi-state analysis of 2006 BRFSS data. Biosecur Bioterror. 2009;7(3):317-330.

5. Aspinwall LG, Sechrist GB, Jones PR. Expect the best and prepare for the worst: anticipatory coping and preparations for Y2K. Motiv Emot. 2005;29(4):357-388.

6. Edwards ML. Social location and self-protective behaviors: implications for earthquake preparedness. Int J Mass Emerg Disasters. 1993;11(3): 293-303.

7. Eisenman DP, Zhou Q, Ong M, et al. Variations in disaster preparedness by mental health, perceived general health, and disability status. Disaster Med Public Health Prep. 2009;3(1):33-41.

8. Mishra S, Suar D. Do lessons people learn determine disaster cognition and preparedness? Psychol Dev Soc J. 2007;19(2):143-159.

9. Murphy ST, Cody M, Frank LB, et al. Predictors of emergency preparedness and compliance. Disaster Med Public Health Prep. 2009 Jul 8 [Epub ahead of print].

10. Norris FH, Smith T, Kaniasty K. Revisiting the experience-behavior hypothesis: the effects of Hurricane Hugo on hazard preparedness and other self-protectiev acts. Basic Appl Soc Psych. 1999;21(1):37-47.

11. Smith DL, Notaro SJ. Personal emergency preparedness for people with disabilities from the 2006-2007 Behavioral Risk Factor Surveillance System. Disabil Health J. 2009;2(2):86-94.

12. Eisenman DP, Wold C, Fielding J, et al. Differences in individual-level terrorism preparedness in Los Angeles County. Am J Prev Med. 2006; 30(1):1-6.

13. Page L, Rubin J, Amlot R, et al. Are Londoners prepared for an emergency? A longitudinal study following the London bombings. Biosecur Bioterror Dec 2008;6(4):309-319.

14. Bethel JW, Foreman AN, Burke SC. Disaster preparedness among medically vulnerable populations. Am J Prev Med. 2011;40(2):139-143.

15. Edwards FL. Effective disaster response in cross border events. Journal of Contingencies and Crisis Management. 2009;17(4):255-265.

16. Strine TW, Neff LJ, Crawford S. Health-related quality of life domains and household preparedness for public health emergencies: behavioral risk factor surveillance system, 2006-2010. Disaster Med Public Health Prep. 2013;7(2):191-200.

17. Boscarino JA, Adams RE, Figley CR, et al. Fear of terrorism and preparedness in New York City 2 years after the attacks: implications for disaster planning and research. J Public Health Manag Pract. 2006;12 (6):505-513.

18. Hausman AJ, Hanlon A, Seals B. Social capital as a mediating factor in emergency preparedness and concerns about terrorism. J Community Psychol. 2007;35(8):1073-1083.

19. Mulilis JP, Duval TS, Rogers R. The effect of a swarm of local tornados on tornado preparedness: a quasi-comparable cohort investigation. J Appl Soc Psychol. 2003;33(8):1716-1725.

20. Sattler DN, Kaiser CF, Hittner JB. Disaster preparedness: relationships among prior experience, personal characteristics, and distress. J Appl Soc Psychol. 2000;30(7):1396-1420.

21. Chen V, Banerjee D, Liu L. Do people become better prepared in the aftermath of a natural disaster? The hurricane Ike experience in Houston, Texas. J Public Health Manag Pract. 2012;18(3):241-249.

22. Morgan C. Hurricane awareness poll results - May 2009. Miami Herald. 2009.

23. Rincon E, Linares MY, Greenberg B. Effect of previous experience of a hurricane on preparedness for future hurricanes. Am J Emerg Med Jul 2001;19(4):276-279.

24. Farley JE. Down but not out: earthquake awareness and preparedness trends in the St. Louis metropolitan area, 1990-1997. Int J Mass Emerg Disasters. 1998;16(3):303-319.

25. Greenberg MR, Dyen S, Elliott S. The public's preparedness: self-reliance, flashbulb memories, and conservative values. Am J Public Health. 2013;103(6):e85-e91.

26. Heller K, Alexander DB, Gatz M, et al. Social and personal factors as predictors of earthquake preparation: the role of support provision, network discussion, negative affect, age, and education. J Appl Soc Psychol. 2005;35(2):399-422.

27. Meyer RJ. Failing to learn from experience about catastrophes: the case of hurricane preparedness. J Risk Uncertain. 2012;45(1):25-50.

28. Centers for Disease Control and Prevention. Deaths associated with Hurricane Sandy - October-November 2012. MMWR Morb Mortal Wkly Rep. 2013;62(20):393-397.

29. Blake ES, Kimberlain TB, Berg RJ, Cangialosi JP, Beven JL. Tropical Cyclone Report - Hurricane Sandy. Miami, FL: National Hurricane Center; 2013.

30. Brackbill RM, Hadler JL, DiGrande L, et al. Asthma and posttraumatic stress symptoms 5 to 6 years following exposure to the World Trade Center terrorist attack. JAMA. 2009;302(5):502-516.

31. Centers for Disease Control and Prevention. Deaths in World Trade Center terrorist attacks-New York City, 2001. MMWR Morb Mortal Wkly Rep. 2002;51(Spec No):16-18.

32. Farfel M, DiGrande L, Brackbill R, et al. An overview of 9/11 experiences and respiratory and mental health conditions among World Trade Center Health Registry enrollees. J Urban Health. 2008;85 (6):880-909

33. Brackbill RM, Caramanica K, Maliniak M, et al. Nonfatal injuries 1 week after hurricane sandy-New York city metropolitan area, October 2012. MMWR Morb Mortal Wkly Rep. 2014;63(42):950-954.

34. Subbarao I, Lyznicki JM, Hsu EB, et al. A consensus-based educational framework and competency set for the discipline of disaster medicine and public health preparedness. Disaster Med Public Health Prep. 2008;2 (1):57-68.

35. Currier M, King DS, Wofford MR, et al. A Katrina experience: lessons learned. Am J Med. 2006;119(11):986-992.

36. Jenkins JL, McCarthy M, Kelen G, et al. Changes needed in the care for sheltered persons: a multistate analysis from Hurricane Katrina. Am J Disaster Med. 2009;4(2):101-106.

37. Brodie M, Weltzien E, Altman D, et al. Experiences of hurricane Katrina evacuees in Houston shelters: implications for future planning. Am J Public Health. 2006;96(8):1402-1408.

38. Torabi MR, Seo DC. National study of behavioral and life changes since September 11. Health Educ Behav. 2004;31(2):179-192.

39. Blanchard EB, Jones-Alexander J, Buckley TC, et al. Psychometric properties of the PTSD Checklist (PCL). Behav Res Ther. 1996;34 (8):669-673.

40. Ruggiero KJ, Del Ben K, Scotti JR, et al. Psychometric properties of the PTSD Checklist-Civilian Version. J Trauma Stress. 2003;16(5):495-502.

41. Ventureyra VA, Yao SN, Cottraux J, et al. The validation of the Posttraumatic Stress Disorder Checklist Scale in posttraumatic stress disorder and nonclinical subjects. Psychother Psychosom. 2002;71 (1):47-53.

42. Debchoudhury I, Welch AE, Fairclough MA, et al. Comparison of health outcomes among affiliated and lay disaster volunteers enrolled in the World Trade Center Health Registry. Prev Med. 2011;53(6):359-363.

43. Welch $\mathrm{AE}$, Caramanica $\mathrm{K}$, Maslow $\mathrm{CB}$, et al. Frequent binge drinking five to six years after exposure to 9/11: findings from the World Trade Center Health Registry. Drug Alcohol Depend. 2014;140:1-7.

44. Ghuman SJ, Brackbill RM, Stellman SD, et al. Unmet mental health care need 10-11 years after the 9/11 terrorist attacks: 2011-2012 results from the World Trade Center Health Registry. BMC Public Health. 2014;14:491.

45. Brackbill RM, Stellman SD, Perlman SE, et al. Mental health of those directly exposed to the World Trade Center disaster: unmet mental health care need, mental health treatment service use, and quality of life. Soc Sci Med. 2013;81:110-114.

46. Adams RE, Boscarino JA, Galea S. Alcohol use, mental health status and psychological well-being 2 years after the World Trade Center attacks in New York City. Am J Drug Alcohol Abuse. 2006;32(2):203-224.

47. New York City Office of Emergency. NYC Hazards: Hurricane Evacuation Zones. 2014. http://www.nyc.gov/html/oem/html/hazards/ storms_evaczones.shtml. Accessed August 21, 2014. 
48. Caramanica K, Brackbill RM, Liao T, et al. Comorbidity of 9/11-Related PTSD and Depression in the World Trade Center Health Registry 10-11 Years Postdisaster. J Trauma Stress. 2014;27(6):680-688.

49. Li J, Brackbill RM, Stellman SD, et al. Gastroesophageal reflux symptoms and comorbid asthma and posttraumatic stress disorder following the 9/11 terrorist attacks on World Trade Center in New York City. Am J Gastroenterol. 2011;106(11):1933-1941.

50. Nair HP, Ekenga CC, Cone JE, et al. Co-occurring lower respiratory symptoms and posttraumatic stress disorder 5 to 6 years after the World Trade Center terrorist attack. Am J Public Health. 2012;102(10): 1964-1973.

51. Faupel CE, Styles SP. Disaster education, household preapredness, and stress responses following Hurricane Hugo. Environ Behav. 1993;25 (2):228-249.

52. Lemyre L, Clement $\mathrm{M}$, Corneil W, et al. A psychosocial risk assessment and management framework to enhance response to CBRN terrorism threats and attacks. Biosecur Bioterror. 2005;3(4):316-330.
53. Rohrmann B. A socio-psychological model for analyzing risk communication processess. Aust J Disaster Trauma Stud. 2000;2:150-166.

54. Cohen S. Psychosocial models of the role of social support in the etiology of physical disease. Health Psychol. 1988;7(3):269-297.

55. Krause N. Chronic financial strain, social support, and depressive symptoms among older adults. Psychol Aging. 1987;2(2):185-192.

56. Levac J, Toal-Sullivan D, O'Sullivan TL. Household emergency preparedness: a literature review. J Community Health. 2012;37(3): 725-733.

57. Paek HJ, Hilyard K, Freimuth V, et al. Theory-based approaches to understanding public emergency preparedness: implications for effective health and risk communication. J Health Commun. 2010;15(4):428-444.

58. Bandura A. Health promotion from the perspective of social cognitive theory. Psychology and Health. 1998;13:623-649.

59. NYC Office of Emergency Management. Know Your Zone. 2014. http:// www.nyc.gov/html/oem/html/get_prepared/know_your_zone/knowyourzone. html. Accessed October 24, 2014. 